\title{
An End-of-life Curriculum: Empowering the Resident, Patient, and Family
}

\author{
JO MARIE REILLY, M.D. and JEFFREY RING, Ph.D.
}

\begin{abstract}
Physician training programs in undergraduate and graduate medical education strongly recommend that their trainees gain experience in helping patients and their families address end-of-life and palliative care issues with knowledge and compassion. Currently these training programs are inadequately meeting this goal. This paper describes a creative 1-day training workshop or several half-day seminars on the end of life, which are delivered as part of our family practice intern orientation. The training includes self-awareness about death, communicating bad news, guidance with paperwork and legal issues, the stages of grief, patient's perspectives on dying, hospice, and physician well-being.
\end{abstract}

\section{INTRODUCTION}

E DUCATION IN END-OF-LIFE (EOL) CARE is imperative for physicians in undergraduate and graduate medical education training programs. Holding physicians accountable for competencies in EOL care is essential in ensuring standardized, quality care for our dying patients. The findings of a recent Association of American Medical Colleges (AAMC) survey of medical school and teaching hospital curricula indicate inadequate EOL teaching, ${ }^{1}$ particularly in the areas of (1) Pain assessment; (2) Pain management; (3) ethics; (4) Physician-patient communication; (5) EOL communication; (6) psychosocial care; (7) personal awareness; (8) bereavement, and (9) EOL clinical experiences.

The AAMC urges residency review committees to include EOL care as part of their required training. In 2000, the Liaison Committee on Medical Education, which accredits medical schools, introduced a requirement that clinical instruction,
". . .must include the important aspects of preventive, acute, chronic, continuing rehabilitation, and end of life care." 2 Questions about EOL care are now being integrated into the National Board of Medical Examiners. ${ }^{3}$ In recent years, efforts have been made to develop guidelines to incorporate palliative care into the multiple levels of primary care education. ${ }^{4}$ The internal medicine and family practice review committees already have guidelines to aid physicians working with dying patients.

The American Academy of Family Practice strongly recommends that all family medicine residents gain experience in helping patients and their families address end-of-life issues with knowledge and compassion. The Academy published Recommended Core Educational Guidelines for Family Practice Residents in End-of-Life Care in $1998,{ }^{5}$ detailing the attitudes, knowledge, and skills that residents will ideally embody by the end of their training. These curricular components include the delivery of bad news, pain man-

Family Practice Residency Program, White Memorial Medical Center, 1720 Cesar E. Chavez Avenue, Los Angeles, California. 
agement, hospice and palliative care, family issues, and the paperwork associated with patient death. ${ }^{6}$ Indeed, Byock ${ }^{7}$ has suggested that it is incumbent on family physicians to fill the current void of EOL care currently available. It is therefore also incumbent on residency programs to develop and deliver comprehensive and compassionate curricula for care at the end-of-life.

EOL issues tend to receive little attention in medical school and residency curricula. In fact, Rabow and colleagues ${ }^{8}$ reviewed EOL care materials presented in 50 medical textbooks from multiple specialities, concluding that "these textbooks generally offered little helpful information on caring for patients at the end of life." The American Medical Association has taken bold steps to remedy the lack of information through their publication of the Education for Physicians on End-of-Life Care (EPEC) Curriculum ${ }^{9}$ and a dedicated edition of the Journal of the American Medical Association in November 2000. ${ }^{10}$ Additionally Academic Medicine dedicated its April 2002 issue to the need for better physician training in EOL care. ${ }^{11}$ We have found all of these to be rich sources of materials to enhance our training program.

Not only do residents require training in caring for the terminally ill, they also benefit from support in facing their own anxieties associated with death and potential death of their patients. ${ }^{12}$ To this end, residents require adequate self-assessment and healthy coping skills to deal with the complex EOL issues they will confront over the course of professional training and practice. Only when a resident is adequately provided with EOL education and tools for self-care, can he/she successfully empower and support terminally ill patients and their families. Faculty members who have not received formal EOL training may also require continuing education and support so as to become excellent models and teachers for their learners. This paper describes the introductory component of our residency program's longitudinal EOL curriculum delivered during one full day of intern orientation, although the activities can be interspersed throughout a longitudinal experience.

Scheduling an entire orientation day dedicated to EOL teaching makes a bold statement about the importance placed on these issues for our residents. A full orientation day allows us to create sufficient time and space to begin a sensitive and comprehensive exploration of a number of related topics in two general areas: (1) the psycho- logical aspects of bereavement (including a personal inventory of resident's EOL attitudes and experiences) and (2) the legal aspects of death (including death pronouncements, organ donation, coroner's laws, etc.). Our day's learning activities reflect a number of teaching modalities, including the use of popular culture to sustain involvement and attention. Imbedded in our curricular objectives for the day (Table 1), is our additional wish to encourage open conversations that facilitate team building among members of the intern class who will be working closely together for the 3-year duration of residency.

The teaching outline for our EOL orientation day is given in Table 2. We begin with an overview of the day and brief introductions of faculty members and interns. The entire day is co-facilitated by a two-person team of medical and behavioral sciences residency faculty members. We ask residents to fill out a pretest of attitudes, knowledge, and skills, and then quickly more to our first EOL awareness exercise.

\section{PART 1. EOL QUESTIONNAIRES AND PHYSICIAN WELL-BEING}

While there are few phenomena more universal than death, we have noticed a large variability in personal experiences with death in family members and/or friends among our residents.

\section{Table 1. End-of-Life Orientation Curricular Objectives}

At the conclusion of the end-of-life orientation, the PGY-I should be able to:

1. Demonstrate the ability to communicate effectively and compassionately with dying patients and their families.

2. Discuss advance directives with patients, and be able to advise patients and families on the documentation of their wishes.

3. Discuss "bad news" with families compassionately, confidently, and appropriately.

4. Demonstrate the knowledge to accurately and confidently "pronounce" patients dead, fill out death certificates, coroner's reports and organ donation forms.

5. Have increased understanding of the role of hospice in end-of-life care.

6. Reflect on their role and limitations as a physician caregiver, including improved self-awareness of burnout, and increased knowledge of coping strategies. 
Table 2. End-Of-Life Orientation Schedule

\begin{tabular}{ll}
\hline $\begin{array}{l}\text { 9:00-9:10 } \\
\text { 9:10-9:20 }\end{array}$ & $\begin{array}{l}\text { Intern and Faculty Introductions and Overview of the Day } \\
\text { Part I }\end{array}$ \\
$\begin{array}{l}\text { P:20-10:00 } \\
\text { Part II }\end{array}$ & End-of-life interviews in pairs/physician well-being \\
10:00-10:15 & Role-play demonstration: Breaking bad news \\
Part III & \\
10:15-10:45 & Didactic presentation: the paperwork of death \\
10:45-11:00 & Break \\
$\begin{array}{l}\text { Part IV } \\
\text { 11:00-11:30 }\end{array}$ & Video viewing and discussion: Steel Magnolias \\
Part V & Lunch and book club discussion: Tuesdays with Morrie \\
11:30-1:30 & Break \\
1:30-2:00 & Interviewing a simulated patient and debriefing or introduction to hospice \\
Part VI &
\end{tabular}

Equally variable are experiences with dying patients during medical school training. A study by A Coalition for Compassionate Care of the dying found that " ... data from professional caregivers indicate a serious lack of professional education in the skills, behaviors, and value of comprehensive, supportive care for persons with life-threatening illness. Despite the frequent interactions they have with such patients and their loved ones, professional caregivers often do not take the time to learn from these experiences."13

Thus, we begin by pairing up our interns and allowing them time to interview each other about EOL issues, according to the Facing Your Own Death questionnaire and the Inventory of Death Experiences provided to them (Tables 3 and 4).
These questions are adapted from the work of the Reverend Alice Parsons Zulli, Certified Thanatologist at the Glendale Adventist Medical Center in Glendale, California. For practical purposes, we have one resident interview his or her partner with the first instrument, and switch to allow the second partner to interview the first according to the second instrument.

At the conclusion of the interviews in pairs, we debrief the experience as a group, inquiring as to what they discovered about themselves and each other. We have been impressed with the depth and openness of these discussions. The intimacy of the pairs is sustained when we talk as a larger group. Interns tell us about their fears of making a mistake that can kill a patient. They describe

Table 3. Reflection Questions: Facing Your Own Death

1. Death to me is ...

2. Dying to me is ...

3. I wish to life to the age of

4. I do not wish to die before...

5. But I do wish to die before ...

6. To me, the most comforting aspect of dying is ...

7. To me, the most frightening aspect of dying is . .

8. I would/would not want to be told that I am dying because . . .

9. In case of a prolonged terminal illness, I would want:

a) All means used to prolong my life

b) Only normal means used so as to allow death to occur in its own time

c) Extraordinary means to shorten my life

10. When thinking of my own death, I will have the hardest time giving up ...

11. I will have the easiest time giving up . . .

12. As concerns life after death, I believe .. .

13. I wish to be remembered by my family as . . .

14. I wish to be remembered by my friends as ...

Adapted from A. Parsons Zulli. 
Table 4. InVentory of Death Experiences

1. The first or most meaningful death I have ever experienced was ...

2. I was years old when the above death occurred

3. At the time I felt ...

4. In relation to that death, I was most curious about ...

5. The thing(s) that most frightened me was/were ...

6. The feelings I have now as I think back on that death are ...

7. The first funeral or memorial service I ever attended was ...

8. The most memorable aspect of that funeral was ...

9. The first personal friend of my own age who has died was...

10. In relation to this death of someone my own age, I recall thinking ...

11. My first experience of death in a professional setting was...

12. That experience affected me in the following ways ...

13. The most traumatic death I have ever experienced was ...

14. The closest near-death experience I have had was ...

Adapted from A. Parsons Zulli.

deaths they have witnessed in their families and/or during medical school, and the cold insensitivity they have observed in medical instructors and colleagues. We return to these questionnaires in one-on-one teaching sessions with the residents 2 years later during their secondyear geriatrics rotations, affording an opportunity to explore how attitudes and experiences with EOL issues may have changed during the intervening period.

This discussion concludes with a group reflection on the stressful nature of facing death by medical professionals trained to heal and cure. A brief didactic presentation links this stress to the risk of physician impairments, emphasizing statistics on prevalence of alcohol, drugs and suicide among physicians. In the spirit of prevention, we invite the interns to share their own current and anticipated coping strategies to sustain well-being when the stresses of residency training begin to rise.

\section{PART II. DELIVERING BAD NEWS}

The faculty members act out a clinical situation in which a physician must deliver bad news about a poor prognosis to a patient. The situation is presented two times, the first in an attempt to demonstrate "what not to do." Using humor and broad stereotypes of a physician who "does everything wrong," we encourage residents to identify the problems in communication they observe (e.g., avoiding clear language, turning away from the patient nonverbally, multiple distractions by pager and phone calls during the conversation). The scenario is repeated with a demonstration of clear, compassionate, respectful communication. Here, too, residents are invited to share observations and impressions, as we begin to articulate a list of helpful interviewing and relationship strategies.

We have also found the EPEC video vignettes to be provocative and stimulating for discussion on physician communication of EOL issues. When time does not permit our using these videos during orientation day, we reserve them for future lectures and presentations. Our group viewing of module number 12, in particular, has generated much discussion (and debate) about the effectiveness with which the physician discusses imminent death with the daughter of a patient in hospice care. We find these spirited discussions to be quite helpful as residents begin to explore and define their own communication styles.

\section{PART III. PAPERWORK AND LEGAL ISSUES}

While our EOL day is interwoven with focus on personal and family considerations in the EOL, we also spend significant time on the legal aspects of death, also known as "the paperwork of death." Here we seek to increase familiarity, comfort, and ability of the residents to accurately fill out the required documents when a patient dies in the hospital.

In a didactic presentation, the faculty reviews death pronouncements, death certificates, coroner's laws, state reporting laws, organ donation, and durable power of attorney for health care (DPAHC). We have found it both challenging and 
engaging to have the participants anonymously fill out their own death certificate and/or DPAHC. This has been done successfully in other institutions ${ }^{14,15}$ and we have used a simplified, focused hospital form (Table 5) when having the interns express their own DPAHC. While the exercise has generated intense personal discussion, it has been perceived as both challenging and provocative. Interestingly, while providing similar continuing medical education (CME) for faculty, we have found them more comfortable filling out the DPAHC form for "case examples" than for themselves. We ask them to indicate DPAHC and donot-resuscitate (DNR) decisions for both a young, practicing physician and an elderly retired family physician. Our debriefing discussion highlights comparisons between the two response sets, and often moves toward personal revelations of struggles with these issues. This exercise has also surfaced and highlighted age stereotypes and shifts in comfort with death when it occurs outside of "the normal life cycle." These experiential exercises provide an in-depth understanding of what the dying process can be like for patients, families and health professionals, as well as the many difficult decisions patients and their families must make along the way.

\section{PART IV. THE STAGES OF GRIEF: STEEL MAGNOLIAS}

We do not spend a great amount of time on the stages of grief during the orientation day, reserving that for future lectures, precepting, and inpatient rounds teaching opportunities. We do, however, use a film clip from popular culture to challenge the interns to identify potential grief and emotional responses to the loss of a loved one. Toward the end of the film, Steel Magnolias, there is a scene at the conclusion of a funeral for her adult daughter, when the character played by Sally Field demonstrates an impressive range of reactions including denial, anger, and sadness. After viewing the film clip, we engage the interns in a discussion of (1) the various emotional reactions observed in this grieving character and (2) the various interpersonal dynamics observed as this woman grieves among friends. Our discussions have also evolved into issues of gender and grief, the role of social support, as well as the importance of spirituality and religion for many individuals at the end of life.

\section{PART V. LIFE THREATENING ILLNESS} AND DEATH: TUESDAYS WITH MORRIE

For the past several years we have initiated a very successful book club model for discussing life-threatening illness and the experience of dying. In the months prior to the start of residency, we send a copy of Tuesdays with Morrie ${ }^{16}$ to all of our incoming interns with a letter asking them to read the book prior to the start of internship, and indicating that we will be discussing their reactions together on their arrival. Tuesdays with Morrie is a moving account of one man's reflections on the dying process (he is a retired college professor with amyotrophic lateral sclerosis [ALS]) as told to a former student who meets with him every Tuesday. The book is remarkable not only for the candor with which death is approached and described, but also for the rich and inspiring description of Morrie, a mindful and fearless charismatic man with a passion for life.

Our book club discussion is open-ended and freewheeling. Interns are typically eager to share their reactions, and are encouraged to read aloud passages from the book that most speak to them. We also ask them to talk about the process of reading the book, their reactions when they first received it, and how it felt to have all read Tuesdays with Morrie in parallel. One resident commented on how the book, overflowing with Morrie's passion for life, presented a big existential challenge for him as he begins the work-intensive internship year that he anticipated would allow for precious little time for diving into a personal life beyond the hospital confines. Thus, this exercise is also valuable in encouraging interns to struggle with resident wellbeing issues, presenting cultural material on death from a Jewish perspective, and contributing to our intern class team-building objective as well. ${ }^{17}$

\section{PART VI. INTERVIEWING A DYING PATIENT: THE CASE OF MR. SOLOMON}

Toward the end of our day, we have our intern group interview an actor playing a standardized patient, Mr. Solomon, who is terminally ill in hospice at his home, and facing an imminent death from lymphoma. The interviewing intern is encouraged to stop the process at any time and ask for group feedback, ideas, and support. The actor and case are made avail- 
Table 5:

\section{DNR Pre-Arrest Protocol \\ Physician Order Sheet}

NOTE: The intent of this form is to identify patient care preferences. It is to be completed by the Attending Physician within 24 hours of DNR order and to be renewed when the patient is transferred to different level of care. For patients in Villa del Sol the order must be renewed every 30 days.

DNR orders apply only to when a patient has cardiac or respiratory arrest-no pulse, no breathing. Any other issues related to level of care are to be ordered separately (see below).

The following questions are important when determining the appropriate DNR Pre-Arrest Protocol for this patient.

Does this patient have a terminal ${ }^{1}$ illness? $^{2}$

Yes

No

Goal of Therapy this Admission: Curative

Palliative

Both

Patient has decision-making capacity?

Yes

No

Patient has Advance Directive? (i.e., DPAHC)

Yes

No

Legal Next-of-Kin or Surrogate Decision-Maker:

Family Spokesperson (if different from above):

Date and Time of "Family \& Attending M.D. Conference"*:

(*should include a family member, patient's nurse, the chaplain and a social worker)

Appropriate level of Care: ICU/CCU____ DOU___ Med/Surg___VDS___Home___ Hospice

'less than 6 months to live
Reference: Bioethical guidelines in Pt Care Manual (i.e. DNR. Withholding and Withdrawing, Brain Death) and Administrative Manual

(i.e. Ethical Issues, Dealing with)

\section{Limitation of Care Orders: (check all that apply)}

\begin{tabular}{|l|l|l|l|}
\hline & No Chest Compressions & & No Invasive Lines (i.e., Swan-Ganz, arterial lines) \\
\hline & No Defibrillation & No IV Fluids \\
\hline & No Intubation & No Anti-arrhythmics \\
\hline & No Mechanical Ventilation & No Antibiotics \\
\hline & No T-Tube & & No Vasopressors (i.e., dopamine) \\
\hline & No Tracheotomy Surgery & No Critical Care Units \\
\hline & No PEG Surgery & No Nutrition / Tube Feedings \\
\hline No Pacemaker & & No Dialysis \\
\hline No ABG's & & Other (specify): \\
\hline No Blood Drawing & & Other (specify): \\
\hline
\end{tabular}

\begin{tabular}{cc}
\hline DATE & TIME \\
& TATE
\end{tabular}


able to us from USC Keck's Medical School's education department.

This exercise is helpful in putting into practice some of the skills and attitudes we have discussed during the day as well as to facilitate intern collaboration on difficult cases. As observing faculty, we also gain an initial evaluation of our intern's interviewing and communication skills. As more of our interns enter our program having worked with standardized patients and EOL experiences in medical school, this may be a curricular component we will look to change or replace with an exposure to hospice care described below.

\section{PART VII. HOSPICE: A TEAM APPROACH}

The residents receive an introduction to the multi-interdisciplinary nature of hospice by attending and observing a hospice team meeting. The hospice physician leader welcomes the interns with a brief overview about hospice and indications for referral. They then listen to the hospice patient cases as they are presented by each member of the interdisciplinary hospice team. Our goal is to expose the interns to hospice early on in their training, particularly when "the only thing that determines the number of patients that die in an institutional setting is the presence of a hospice in a community." 18

\section{PART VIII. “THE TURNING POINT": A REFLECTIVE WRITING EXERCISE}

As a group, we read the article "The Turning Point"19 aloud. We have chosen this article, written from a physician's perspective, as a rich counterpoint to the patient's perspective on dying explored both through the Mr. Solomon case and Tuesdays with Morrie. After reading the article, we have an open-ended discussion of the intern's impressions and reflections on the essay. They are encouraged to share personal experiences that the article reminds them of, and to note which portions of the essay feel familiar and/or unfamiliar to them based on past experience. Next, we ask each resident to choose one of the individuals described in the essay, and to continue to write in the voice of that individual. The specifics of the writing task are unstructured, allowing the interns a free hand at the assignment. We encourage them to write without self-censure and to take risks. Next, we each read our paragraphs and share impressions. This activity moves the residents to engage a creative, expressive aspect of themselves, encourages empathy, and sets an example of the type of mindful attention to the human drama around them that we hope they will sustain during their years of training and beyond. ${ }^{20}$

\section{CONCLUSIONS}

This paper reviews a comprehensive and creative approach to teaching EOL care in a family practice residency program. Specifically, it details an intensive, one-day seminar for new interns. Residency programs that do not have the luxury of dedicating a full day to this topic in internship, may consider breaking it into several half-day seminars. We have found, however, that the intense and emotionally charged nature of this topic demands larger blocks of time and processing space to be most successful. We also have found that introducing EOL topics early on in training provides a foundation of knowledge, communication skills, and coping strategies that then empower the resident in their future training. Providing a time for reflection on self-care and physician limitations is particularly important for nurturing well-being and preventing burnout and cynicism.

We have also implemented a variety of teaching modalities to include popular culture, books, videos, poetry, music, personal reflections and surveys. The creative and interactive multimedia seminar helps increase accessibility to a difficult and challenging topic, and emphasizes the pervasive nature of end-of-life issues in our communities and larger culture.

Our residency training in EOL is strengthened by our providing continuing education for our faculty. As such, through residency retreats and periodic lectures, we hope to continue to stimulate discussion and "train our trainers" as excellent faculty role models. Teaching about the EOL has traditionally fallen under the rubric of behavioral sciences training. While a psychological perspective is a critical component of EOL curriculum, it is imperative to have a medical faculty as a role model in the teaching process. The latter's direct experience with death; case exam- 
ples and legal knowledge augment the behaviorist's expertise. Furthermore, the medical doctor's active teaching presence sends a strong message about the value we place on the EOL and palliative care components of our curriculum. Additionally, as we expand our EOL curriculum, we are hoping to expand our resource library, as well as looking to include a seminar on spiritual and cultural differences in bereavement and EOL using music, poetry and literature.

Finally, as this curriculum has now been in place several years, the authors have been collecting pretest and posttest data on resident EOL knowledge, attitudes and skills both at internship and at residency graduation. Outcome data will be forthcoming in a future paper. This latter paper will address the strength of our EOL curriculum in preparing our residents to best serve our dying patients and their families.

\section{REFERENCES}

1. Palliative and end-of-life care: a Course requirement. AAMC Reporter 1999;9(3):3-5.

2. Liason Committee on Medical Education: Functions and structure of a medical school. 2000. <www.lcme. org/standard.htm $>$ (Last accessed on January 2, 2002).

3. Bowles T. USMLE and end of life care. J Palliat Med 1999;2:3-4.

4. Block SD, Bernier GM, Crawley LM, Farber S, Kuhl D, Nelson W, O’Donnell J, Sandy L, Ury W: Incorporating palliative care into primary care education: Principles, challenges and opportunities for the National Consensus Conference on Medical Education for Care Near the End of Life. J Gen Intern Med 1998; 13:768-773.

5. American Academy of Family Practice: Core Educational Guidelines for Family Practice Residents in End-ofLife Care, 1998.

6. Wagner W. Teaching interns and residents about death and dying. J Am Osteopath Assoc 1993;93:611613.

7. Byock R: Dying Well: The Prospect for Growth at the End of Life. New York, NY: Riverhead, 1997.
8. Rabow NW, Hardie GE, Fair JM, McPhee SJ: End-oflife care content in 50 textbook from multiple specialties. JAMA 2000;283:771-778.

9. American Medical Association: Education for Physicians on End-of-life Care: Trainer's Guide. Chicago: American Medical Association, 1999.

10. American Medical Association. JAMA 1999;284:24132550.

11. Weissmann DE, Block SD: ACGME requirements for end-of-life training in selected residency and fellowship programs: A status report. Acad Med 2002; 77:299-304

12. Dozor RB, Addison RB: Toward a good death: An interpretive investigation of family practice residents' practices with dying patients. Fam Med 1992;24: 538-543.

13. McSkimming S: Living and Healing During Lifethreatening Illness. Portland, OR: Supportive Care of the Dying: A Coalition for Compassionate Care. 1997.

14. Myers CA, Farquar RE: Improving the accuracy of death certification. CMAJ 1998;158:1317-1323.

15. Simpson M. Do-it-yourself death certificate in evoking and estimating student attitudes toward death. J Med Educ 1975;50:475-478.

16. Albom M: Tuesdays with Morrie. New York, NY, Bantam Doubleday Publishing Group, 1997.

17. Ring, JM, Reilly, JM: Tuesdays with Morrie: A humanities teaching exercise in palliative and end-of-life care. Family Medicine 2003;35:552-554.

18. SUPPORT Principal Investigators: A controlled trial to improve care for seriously hospitalized patients: the Study to Understand Prognoses and Preferences for Outcomes and Risks of Treatment (SUPPORT). JAMA 1995;274;1591-1598.

19. Corrington K: The turning point. Family Med 2002; 34:245-246.

20. Reilly, JM, Ring, JM: Innovations in teaching: Turning point. Family Medicine 2003;35:474-475

Address reprint requests to: Jo Marie Reilly, M.D. Family Practice Residency Program White Memorial Medical Center 1720 Cesar Chavez Avenue Los Angeles, CA 90033

E-mail: reillypas@aol.com 